\title{
Functional appliances and mandibular growth - is there an effect?
}

\author{
Do functional appliances enhance mandibular growth in the treatment of \\ skeletal Class II malocclusions?
}

\author{
Chen JY, Will LA, Niederman R. Analysis of efficacy of functional \\ appliances on mandibular growth. Am J Orthod Dentofacial \\ Orthop 2002; 122:470-476
}

Data sources A MEDLINE search strategy for the years 1966 to 1999 was developed. The search was limited to randomised controlled trials (RCT) and meta-analyses, which were performed on humans and written in English.

Study selection Articles retrieved were critically appraised for their validity. For inclusion, an article had to meet four of seven validity standards and also the following criteria: it pertained to functional appliance use in the early treatment of Class II malocclusions; it was an RCT; and it included measurable mandibular cephalometric values.

Data extraction and synthesis Cephalometric values were used to assess mandibular growth in the horizontal and the vertical dimensions. The following linear measures were assessed: condylion-pogonion (Co-Pg), articulare-pogonion (Ar-Pg), condylion-gnathion (Co-Gn), articulare-gnathion (Ar-Gn), sella-gonion ( $\mathrm{S}-\mathrm{Go})$, articulare-gonion (Ar-Go), and condylion-gonion (Co-Go). Two angular parameters, sella-nasion-B point (SNB) and lower incisal angle (LIA), were also measured. Three horizontal measurements were also used in some of the studies examined, namely: gonion-menton (Go-Me), pogonion- $\mathrm{N}$ $(\mathrm{Pg}-\mathrm{N})$, and gonion-pogonion (Go-Pg). Annualised changes (linear or angular) in specific cephalometric data were pooled from the selected articles and then compared and plotted. Statistical significance was tested through analysis of variance, the Student's $t$-test for paired data and $95 \%$ confidence intervals.

Results The search identified 17 articles of which six met the inclusion and validity criteria. There was a significant difference between the control and the treated groups for $\mathrm{Ar}-\mathrm{Pg}$ and $\mathrm{Ar}-\mathrm{Gn}$. No other horizontal or vertical cephalometric measurements were statistically significant.

Conclusions It is currently difficult to obtain definitive answers about efficacy of functional appliances on mandibular growth because of many inconsistencies in measuring the treatment outcome variables. There is still a need to conduct more RCT to reduce the methodological limitations.

\section{Commentary}

Perhaps this question has divided orthodontic opinion more than any other in recent decades. Although the answer is becoming clearer, articles such as this can be particularly useful to clinicians in providing a synopsis of the best available evidence in this area. Clinicians who use functional appliances know that they correct Class II malocclusions effectively, if worn well.

The description of the method in this review is clear, but it is interesting that the search strategy for RCT used Medline only. There is some evidence that the use of a single-search strategy may not unearth all relevant studies, and searching the main journals by hand, perhaps covering a period of the last 10 years, would have been an improvement. On the other hand, the authors have included all of the major studies carried out during the 1990s, and it is evident that there are few, if any, RCT related to functional appliance therapy before this time. In the end, a search of over 23000 orthodontic articles yielded only six trials that met all the inclusion criteria. The authors then annualised the cephalometric changes that took place in both the treated and control groups. They found that for most measurements of mandibular length change there was no statistically significant difference between treated and untreated patients, except for two variables, Ar-Gn and $\mathrm{Ar}-\mathrm{Pg}$. For these, there was a statistically significant difference between the treated and control groups. The authors concluded, however, that movement of articulare during treatment with functional appliances, allied with the difficulty in landmark identification of articulare (and therefore an increase in the error of the method), reduces the significance of this result.

The article does not come to any firm conclusion as to whether functional appliance therapy increases mandibular growth. As is often the case, the only real conclusion is that there is still a need to conduct more randomised trials to reduce the methodological limitations. Since this article was written, one RCT has concluded that a functional appliance does not, on average, change a child's Class II skeletal pattern to any significant degree, at least in the case of early treatment. ${ }^{1}$

\section{Practice point}

- Functional appliances can correct significant Class II malocclusions, but this study was unable to conclude whether functional appliances enhance mandibular growth.

\section{Julian RS O'Neill \\ Consultant Orthodontist, Kettering General Hospital NHS Trust, Kettering, Northamptonshire, UK}
1. O'Brien K, Wright J, Conboy F, et al. Effectiveness of early orthodontic treatment with a twin-block appliance: a multi-centre randomised control trial. Part 1 Dental and skeletal effects. Am J Orthod Dentofacial Orthop 2003; 124:234-243.

Evidence-Based Dentistry (2004) 5, 74.

doi:10.1038/sj.ebd.6400268
Address for correspondence: Leslie A Will, Department of Growth and Development Harvard School of Dental Medicine, 188 Longwood Avenue, Boston MA 02115, USA E-mail: Iwill@hsdm.harvard.edu 